Ann. Zootech., I972, 21 (3), 45I-460.

\title{
ENREGISTREMENT GRAPHIQUE DES CONSOMMATIONS D'ALIMENTS SOLIDE ET LIQUIDE DU LAPIN DOMESTIQUE NOURRI AD LIBITUM
}

\author{
M. PRUD'HON, Y. CARLES, J. GOUSSOPOULOS et P. F. KOEHL \\ Station de Physiologie animale, \\ École nationale supérieure agronomique, I. N.R. A., \\ Place Viala, \\ 34 - Montpellier
}

\section{RÉSUMÉ}

Nous décrivons un dispositif d'enregistrement graphique automatique des consommations d'aliments solide et liquide du Lapin domestique nourri ad libitum.

Ce dispositif a été utilisé pour enregistrer la consommation de 6 lapins issus d'une même portée, 3 mâles et 3 femelles, âgés de 28 à 34 semaines.

Les sujets étudiés ont fait, en moyenne, 23 à 33 repas solides par jour, d'une durée totale de $I \mathrm{~h}$ 30 à $2 \mathrm{~h} I 5$ et $\mathrm{I} 3$ à I9 repas liquides.

Le nombre, la durée et la répartition des repas au cours des 24 heures sont relativement répétables d'un jour à l'autre chez le même individu mais diffèrent sensiblement d'un individu à l'autre.

60 p. Ioo environ des consommations d'eau et d'aliment granulé ont lieu au cours des Io heures d'obscurité ; cette inégalité de répartition est due à une plus grande fréquence des repas nocturnes et à l'existence d'un " repos alimentaire " plus ou moins prolongé au cours de la période d'éclairement.

\section{INTRODUCTION}

La consommation d'aliments est sans doute le facteur qui agit le plus directement sur la production laitière des lapines et la vitesse de croissance des lapereaux et, par conséquent sur leur indice de consommation (PRUD'HON, I967; LEBAS, Ig69 $a$; ROUVIER, I970). Il est donc important de bien connaître les particularités du comportement alimentaire $\mathrm{du}$ lapin afin de pouvoir contrôler sa croissance.

Si l'on met à part les nombreux travaux ayant porté sur l'ingestion des cæcotrophes (cf. revue de WORDEN et LeAHy, I962 : FAURE, Vincen'T et Bensch, Ig66 ; LEBAS, 
I969 b), on a peu de renseignements précis sur le comportement alimentaire du lapin. On sait que pendant l'allaitement, les lapereaux ne sont admis à téter qu'une ou deux fois par jour en moyenne (ZARROW, DENENBERG et ANDERSON, I965 ; YARDIN, I 968) ; par contre, après le sevrage qui peut être réalisé très précocement (PRUD'Hon et BEL, I968), ils consomment des quantités importantes de matière sèche (jusqu'à Io p. Ioo de leur propre poids par jour) au cours de très fréquents repas.

L'adaptation à cette nouvelle répartition des prises d'aliments paraît rapide mais n'a pas été étudiée $\in$ n détail.

On sait également que le Lapin nourri ad libitum consomme la plus grande partie de sa ration durant la nuit ( 2 tiers à 3 quarts des quantités ingérées chaque jour selon PRUD'HON (I967) et LEBAS (I970). Le temps consacré à la prise d'aliments est assez mal connu; il serait de 4 à 6 heures (LEBAS, I970) avec probablement des variations importantes; ainsi la croissance de lapins à l'engraissement n'est pas ralentie s'ils ne disposent que d'un poste d'alimentation par groupe de dix (LEBAS, I97I).

Compte tenu de l'imprécision des données existantes, il nous a paru utile de mettre au point un dispositif d'enregistrement graphique des phases de prise d'aliments et de repos alimentaire du Lapin. Nous décrivons ici ce dispositif et donnons des résultats concernant la fréquence, la durée, l'importance et la répartition dans le temps des repas enregistrés chez des lapins des deux sexes, en fin de croissance, qui ont servi à tester le fonctionnement de ce dispositif.

\section{MATÉRIEI, E'T MÉTHODES}

\section{I. - Dispositif d'envegistrement}

Le dispositif d'enregistrement est schématisé sur la figure I.

Une trémie tarée est placée à demeure sur le plateau d'une balance automatique sensible au gramme près ; un stylet, fixé directement sur l'aiguille de la balance, inscrit de façon continue sur le papier de l'enregistreur le poids des réserves d'aliments.

Sur le même papier sont enregistrées les consommations d'eau grâce à un stylet, solidaire d'un flotteur placé dans le réservoir de l'abreuvoir à clapet qui alimente en eau le lapin.

Le papier enregistreur est enroulé sur un tambour entraîné par un motoréducteur synchrone ; la vitesse de défilement du papier est de $3 \mathrm{~cm}$ par heure, ce qui permet une précision de l'ordre de la minute dans l'estimation de la durée des repas et des intervalles entre repas.

L'aliment granulé consommé est mesuré au gramme près, l'eau à $2 \mathrm{ml}$ près.

Les lapins soumis au contrôle sont élevés dans des cages individuelles grillagées mesurant $74 \times 52 \times 40 \mathrm{~cm}$. Elles sont suspendues à un bâti métallique par des fils d'acier et oscillent très légèrement lorsque l'animal bouge ou se déplace ; cette activité motrice peut être enregistrée grâce à un dispositif décrit par BAcou, (1970).

\section{2. - Conditions expérimentales}

Les cages sont situées dans une salle maintenue à température constante : $20^{\circ} \pm \mathrm{I}^{\circ} \mathrm{C}$.

L'éclairement, d'une intensité de I ooo lux au niveau des cages, est assuré par des tubes fluorescents de type lumière du jour ; la durée de l'éclairement est réglée par une horloge.

$\mathrm{Au}$ cours de nos premiers travaux nous avons enregistré la consommation d'aliments de 6 lapins en fin de croissance, 3 mâles et 3 femelles issus d'une même portée et appartenant à la souche "commune "élevée au laboratoire.

Ces animaux dont le rythme d'activité motrice avait été enregistré au cours de la croissance (BACOU, I970) étaient parfaitement acclimatés au type de cage et au local d'expérimentation. Ils étaient âgés de 6 mois et demi au début de la période de contrôle alimentaire.

La durée d'enregistrement continu a varié de 5 à 8 jours selon les individus; l'aliment dis- 
tribué était un granulé commercial contenant I5 p. Ioo de matières protéiques brutes et I 2,5 p. Ioo de matières cellulosiques ( $\left.{ }^{1}\right)$.

La photopériode est restée constante pendant tout l'essai : I4 heures d'éclairement, de 7 h à $2 \mathrm{I}$ h, et ro heures d'obscurité.

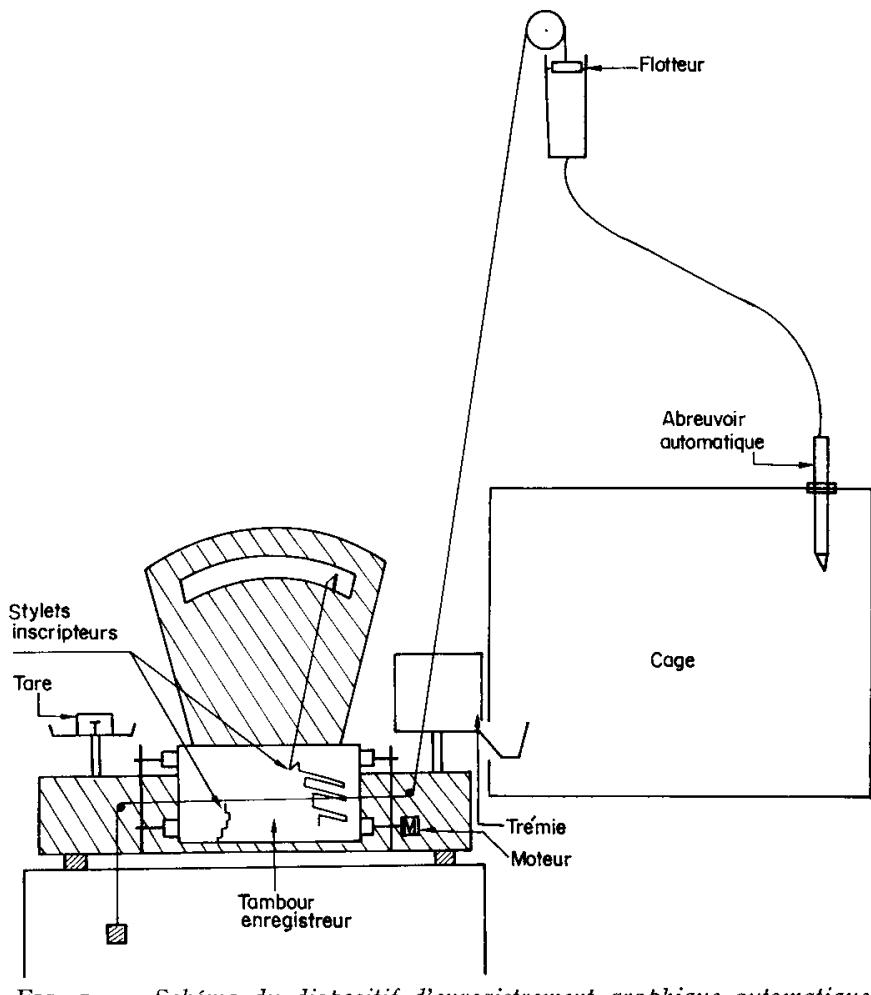

FIG. I. - Schéma du dispositif d'enregistrement graphique automatique des prises d'aliments solide et liquide

\section{RÉ,SULTATS}

\section{I. - Caractéristiques globales de la consommation d'aliments}

Nous présentons sur la figure 2 un exemple des tracés enregistrés pour le granulé et la boisson au cours d'une période de 24 heures.

Les granulés sont consommés au cours d'une succession de repas de durée variable nettement séparés les uns des autres par des paliers plus ou moins longs, les intervalles les plus longs étant toujours situés pendant la photopériode claire; la consommation est donc discontinue et ne présente pas, en apparence, de cycles réguliers au cours des 24 heures.

Les prises de boisson, généralement moins fréquentes, sont d'une faible durée ; leur répartition est également acyclique. Nous n'avons constaté aucune régularité dans l'alternance des consommations de matière sèche et d'eau.

(1) Cofna * Lapins *. 


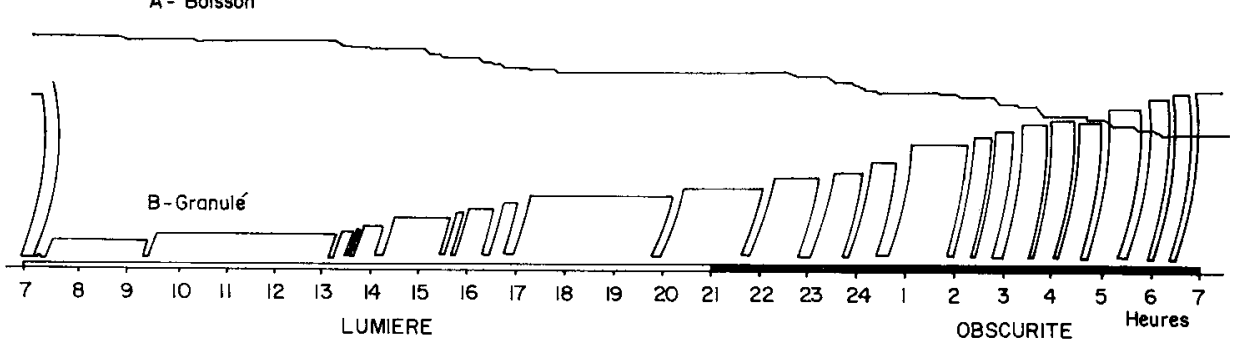

FIG. 2. - Exemple schêmatique de courbes cumulatives de la consommation d'eau et de granulé au cours d'une période de 24 heures

Les courbes A et B représentent respectivement la consommation d'eau (abaissement du flotteur dans le réservoir) et la consommation de granulé (allègement de la trémie). Lorsqu'un lapin consomme du granulé il appuie sur la trémie ; le plateau de la balance s'abaisse et reste au niveau inférieur pendant toute la durée du repas qui peut ainsi être mesurée. Après le repas, le plateau remonte jusqu'au niveau d'équilibre; la quantité consommée est mesurée par l'écart entre deux paliers successifs.

Sur le tableau I sont rassemblées quelques valeurs numériques des caractéristiques de la consommation journalière des 6 lapins soumis au contrôle ainsi que leur poids moyen et leur évolution pondérale au cours de la période d'enregistrement. Malgré la parenté étroite des sujets étudiés et l'identité des conditions d'élevage, il existe des différences entre individus très marquées :

\section{TABLEAU I}

Principales caractéristiques de la consommation journalière d'aliments solides et liquides et de l'évolution pondérale de six lapins en fin de croissance

\begin{tabular}{|c|c|c|c|c|c|c|c|}
\hline & & \multicolumn{3}{|c|}{ Mâles } & \multicolumn{3}{|c|}{ Femelles } \\
\hline & & $110^{\prime}$ & 1105 & 1107 & 1101 & 1102 & 1106 \\
\hline \multicolumn{2}{|c|}{ Nombre de jours de contrôle } & 5 & 6 & 4 & 6 & 5 & 8 \\
\hline \multicolumn{2}{|c|}{$\begin{array}{l}\text { Poids moyen au cours } \\
\text { de la période de contrôle (g) }\end{array}$} & 4050 & 4135 & 1855 & 4745 & 5130 & 4510 \\
\hline \multicolumn{2}{|c|}{ Gain moyen quotidien $(\mathrm{g} / \mathrm{j})$} & +6 & +16 & +4 & -10 & +15 & +14 \\
\hline \multirow{4}{*}{$\begin{array}{l}\text { Aliment solide } \\
\text { (Moyenne } \\
\pm \text { écart-type) }\end{array}$} & $\begin{array}{c}\text { Quantité totale } \\
(\mathrm{g})\end{array}$ & $185 \pm 15$ & $158 \pm 17$ & $252 \pm 14$ & $133 \pm 18$ & $203 \pm 15$ & $212 \pm 16$ \\
\hline & $\begin{array}{c}\text { Durée totale } \\
\text { des repas }(m n)\end{array}$ & $96 \pm 7$ & $100 \pm 11$ & $112 \pm 8$ & $93=14$ & $127 \pm 16$ & $115 \pm 13$ \\
\hline & Nbre repas/jour & $27,8 \pm 3,2$ & $23,0 \pm 3,2$ & $28,3 \pm 2,1$ & $24,7 \pm 2,6$ & $33,0 \pm 3,4$ & $25,9 \pm 3,5$ \\
\hline & $\begin{array}{l}\text { Poids moyen } \\
\text { des repas (g) }\end{array}$ & $6,7 \pm 0,4$ & $7,0 \pm 0,7$ & $9,0 \pm 0,9$ & $5,4 \pm 0,6$ & $6,2 \pm 0,6$ & $8,3 \pm 0,8$ \\
\hline \multirow{3}{*}{$\begin{array}{c}\text { Boisson } \\
\text { (moyenne } \\
\pm \text { écart-type) }\end{array}$} & $\begin{array}{l}\text { Quantité } \\
\text { totale/jour (g) }\end{array}$ & $232 \pm 18$ & $172 \pm 35$ & $294 \pm 42$ & $134 \pm 15$ & $291 \pm 43$ & $276 \pm 26$ \\
\hline & Nbre prises/jour & $18,4 \pm 1,1$ & $15,8 \pm 1,7$ & $17,3 \pm 1,5$ & $13,0 \pm 1,5$ & $19,2 \pm 2,6$ & $14,6 \pm 1,6$ \\
\hline & $\begin{array}{l}\text { Poids moyen } \\
\text { des prises }(g)\end{array}$ & $12,8 \pm 1,5$ & $10,7 \pm 1,4$ & $16,4 \pm 1,4$ & $10,7 \pm 2,2$ & $15,2 \pm 1,5$ & $18,9 \pm 0,8$ \\
\hline
\end{tabular}


La consommation d'aliments varie du simple au double selon les individus :

de $133 \mathrm{~g}$ à $252 \mathrm{~g}$ par jour pour le granulé

de $134 \mathrm{~g}$ à $294 \mathrm{~g}$ par jour pour l'eau.

Il est possible que ces différences soient partiellement liées au poids ou aux variations de poids des sujets ; cela n'apparaît pas nettement sur le tableau I, si l'on excepte le cas de la femelle IIOI qui a peu mangé, peu bu et a maigri.

La durée totale des repas varie de 93 à 127 minutes par jour ;

Le nombre moyen de repas solides de 23,0 à 33,0 ;

Le nombre de prises de boisson de I3,0 à I 9,2 ;

Enfin, le poids moyen de chaque prise est également variable : 5,4 à 8,9 g par repas moyen pour les granulés, 10,3 à I8,9 g pour la boisson.

En revanche, ces différents paramètres varient peu d'un jour à l'autre pour le

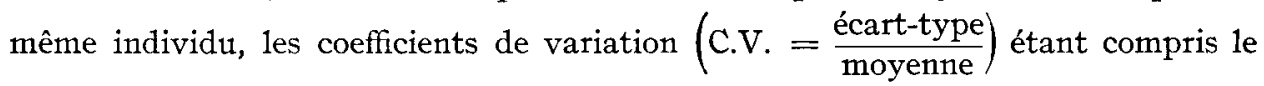
plus souvent entre 0,07 et $0, I_{4}$.

\section{2. - Répartition de la consommation au cours des 24 heures}

Nous avons analysé séparément les consommations réalisées pendant la photopériode claire et la photopériode obscure (tabl. 2).

TABLEAU 2

Répartition de la consommation pendant la période d'obscurité et la période d'éclairement

\begin{tabular}{|c|c|c|c|c|c|c|c|c|}
\hline & & \multicolumn{4}{|c|}{ Mâles } & \multicolumn{3}{|c|}{ Femelles } \\
\hline & & & $110_{4}^{\prime}$ & 1105 & 1107 & 1101 & 1102 & 1106 \\
\hline \multirow{2}{*}{$\begin{array}{c}\text { P. } 100 \text { de } \\
\text { consom. } \\
\text { au cours } \\
\text { des } 10 \mathrm{~h} \\
\text { d'obsc. }\end{array}$} & Granulé & & 60,0 & 66,1 & 58,7 & 58,7 & 57,8 & 49,8 \\
\hline & Boisson & & 65,6 & 66,5 & 62,1 & 67,7 & 64,7 & 46,1 \\
\hline \multirow{4}{*}{$\begin{array}{l}\text { Aliment } \\
\text { solide }\end{array}$} & Nbre moyen & Jour & 0,7 & 0,5 & 0,7 & 0,7 & 1 & 0,8 \\
\hline & & Nuit & 1,8 & 1,6 & 1,8 & 1,4 & 1,8 & $1, \mathbf{k}$ \\
\hline & Importance & Jour & $7,1 \pm 2,8$ & $7,6 \pm 2,3$ & $10,3 \pm 4,7$ & $5,1 \pm 2,3$ & $5,9 \pm 2,2$ & $8,8 \pm 4,5$ \\
\hline & des repas (g) & Nuit & $6,0 \pm 3,3$ & $6,7 \pm 2,8$ & $8,3 \pm 4,0$ & $5,6 \pm 2,4$ & $6,5 \pm 2,5$ & $7,8 \pm 4,0$ \\
\hline \multirow{4}{*}{ Boisson } & \multirow{2}{*}{$\begin{array}{c}\text { Nbre moy. de } \\
\text { prises/h }\end{array}$} & Jour & 0,5 & 0,4 & $0, \bar{a}$ & 0,5 & $0, \overline{5}$ & 0,5 \\
\hline & & Nuit & 1,2 & 1,0 & 1,0 & 0,8 & 0,8 & 0,7 \\
\hline & \multirow{2}{*}{$\begin{array}{c}\text { Importance } \\
\text { des prises }(g)\end{array}$} & Jour & $13,1 \pm 6,4$ & $9,7 \pm 3,2$ & $16,1 \pm 10,1$ & $8,9 \pm 3,2$ & $13,0 \pm 6,3$ & $19,7 \pm 8,1$ \\
\hline & & Nuit & $12,8 \pm 5,1$ & $11,2 \pm 4,4$ & $17,7 \pm 8,5$ & $11,6 \pm 4,8$ & $17,7 \pm 9,3$ & $19,6 \pm 7, \mathbf{x}$ \\
\hline
\end{tabular}


5 des animaux étudiés sur 6 consomment près de 60 p. Ioo du granulé et plus de 60 p. roo de la boisson pendant les ro heures d'obscurité ; le sixième consomme pratiquement, la moitié de sa ration journalière pendant le même laps de temps. Par conséquent tous ces individus ont une consommation moyenne horaire plus élevée de nuit que de jour. Cette différence n'est pas due à l'ingestion de repas plus importants pendant la phase d'obscurité (cf. tabl. 2) : certains sujets effectuent des prises de granulé plus importantes de jour, d'autres boivent davantage à l'obscurité mais il s'agit vraisemblablement de variations aléatoires compte tenu de la variabilité de l'importance unitaire des prises $(0,35<$ C.V. $<0,60)$.

Par contre, le nombre moyen horaire de repas est environ deux fois plus élevé de nuit que de jour chez tous les individus (tabl. 2).

LUMIERE

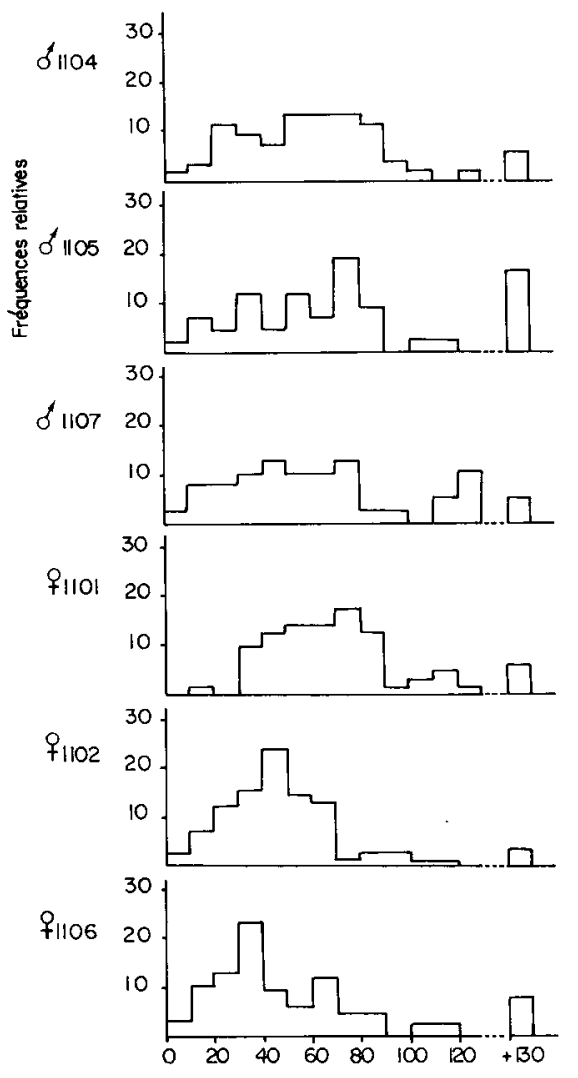

OBSCURITE

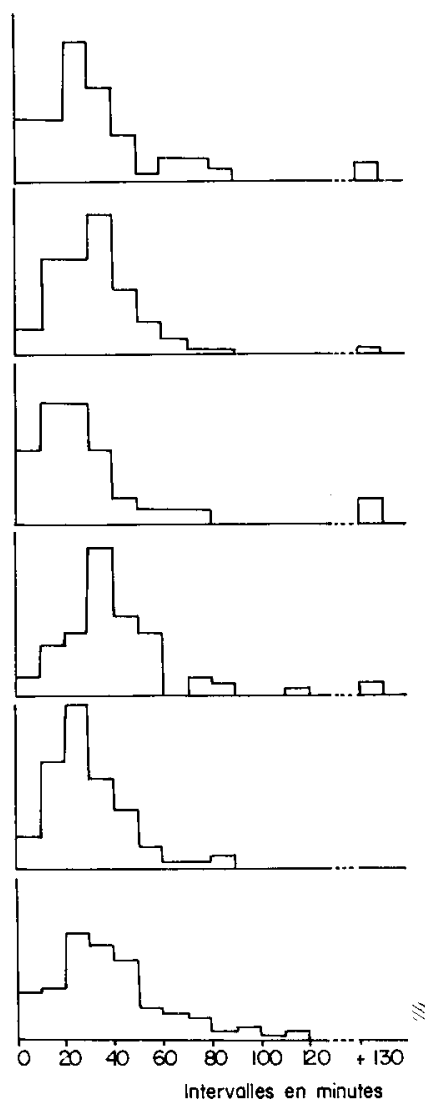

FIG. 3. - Polygones de fréquence des intervalles entre repas successifs de six lapins en fin de croissance au cours des périodes d'éclairement et d'obscurité

L'étude des polygones de fréquence des intervalles entre repas successifs (fig. 3) met en lumière des différences entre la phase d'obscurité et la phase d'éclairement.

De nuit les intervalles sont peu dispersés ; le mode est situé entre 20 et 40 minutes selon les individus ; à la lumière, les intervalles sont très dispersés, notamment chez 
les mâles, le mode diffère d'un individu à 1'autre mais reste toujours supérieur à ce qu'il est la nuit.

La figure 3 met encore en évidence l'allure dissymétrique de la distribution des intervalles, qui s'apparenterait davantage à une distribution de Poisson qu'à une distribution normale.
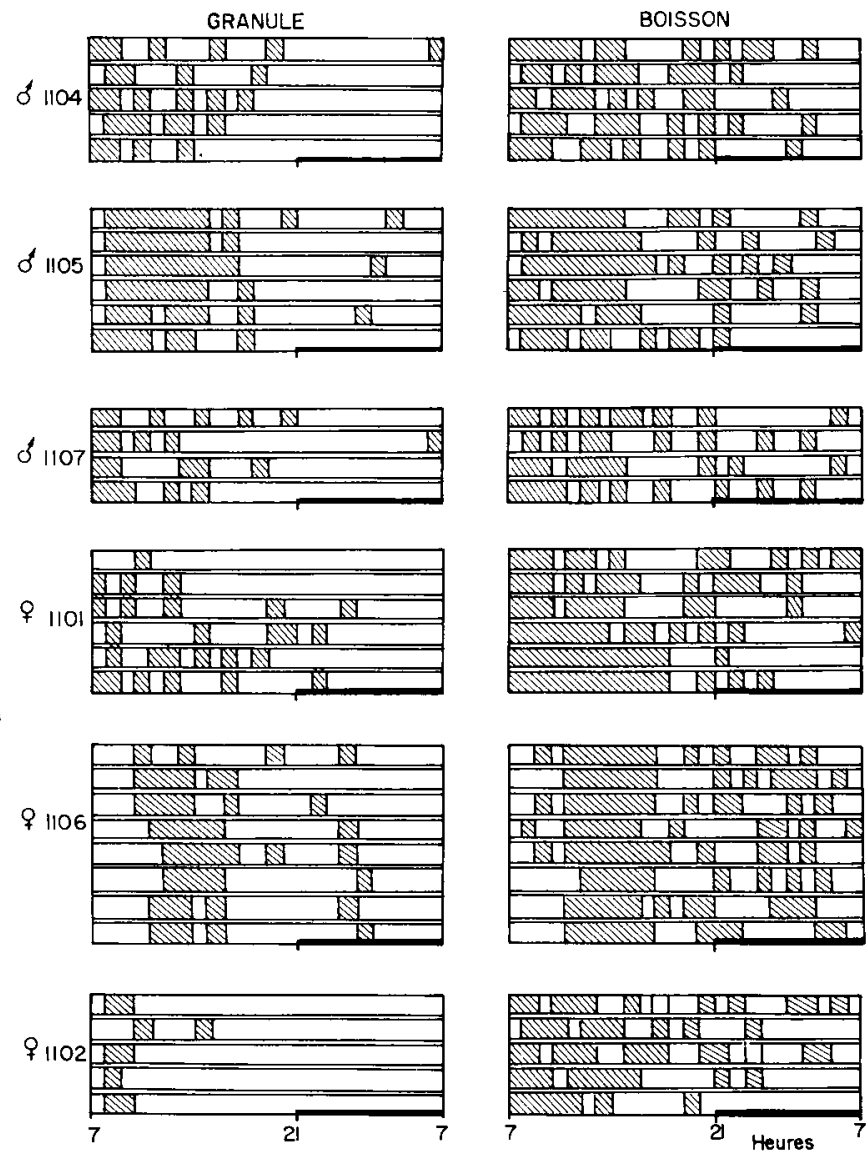

FIG. 4. - Répartition des périodes horaires de consommation d'aliments et de repos alimentaire au cours des séquences d'enregistrement des consommations de six lapins en fin de croissance

Mepos alimentaire

Consommation

Le nycthémère est divisé en 24 périodes horaires ; chaque période est représentée en blanc ou en noir selon que l'animal y a effectué ou non des prises de nourriture.

Chaque ligne représente une journée d'enregistrement; le trait renforcé indique la période d'obscurité (2I h-7 h).

La figure 4 , où sont représentées, heure par heure, les périodes de consommation ou de non consommation d'aliments, met en évidence, chez tous les sujets, l'existence d'une période quotidienne de repos alimentaire plus ou moins continue et prolongée qui se répète assez régulièrement d'un jour à l'autre.

Elle met également en évidence l'existence de variations entre individus de comportement quant à l'heure à laquelle commence ce repos et quant à sa durée : 
Le plus souvent les lapins s'arrêtent de boire et de manger dès la première ou la seconde heure d'éclairement ; la femelle r ro6 attend la quatrième ou la cinquième heure ; le mâle II07 et la femelle I 106 s'arrêtent de manger très longtemps (5 à 8 heures consécutives) alors que la femelle I Ioz ne s'interrompt jamais plus de deux heures par jour.

Enfin, nous avons étudié graphiquement les relations existant entre le poids de chaque repas d'aliment granulé et la durée de l'intervalle qui sépare ce repas du précédent ou du suivant. Dans les deux cas nous avons obtenu des nuages de points extrêmement dispersés permettant de penser qu'il existe peu de relations entre les paramètres étudiés.

\section{DISCUSSION ETT CONCLUSIONS}

Le dispositif d'enregistrement de la consommation d'aliments mis au point au laboratoire a été testé pendant un mois et demi sur 6 sujets différents. Les informations recueillies ont été régulières et précises en ce qui concerne la consonmation de granulés, un peu moins complètes pour la boisson dont nous n'avons pu mesurer la durée des prises.

L,es enregistrements réalisés ont mis en évidence 1'existence de repas solides et liquides nettement individualisés et irrégulièrement espacés dont le nombre, la durée et la répartition au cours des 24 heures sont relativement répétables, d'un jour à l'autre, chez le même individu, mais varient de façon importante d'un individu à l'autre. Nous n'avons donc pas observé un seul comportement stéréotypé pour des individus choisis aussi proches les uns des autres que possible - même portée, conditions d'élevage identiques, même stade physiologique.

En l'absence de données précises concernant le Irapin, ces résultats sont à rapprocher des résultats enregistrés sur des rats blancs adultes par LE MAGNEN et TALION (Ig66) qui constatent " un asynchronisme fondamental de la succession des repas sur des périodes brèves, mais une forte constance du nombre quotidien de repas associée à une constance relative de la consommation totale quotidienne de chaque individu ". Ces auteurs soulignent également "l'absence d'un pattern de prise alimentaire stéréotypé et 1'existence de patterns individuels plus ou moins reproduits d'un jour à l'autre ".

Le caractère "nocturne " de la consommation d'aliments du Lapin a été confirmé, les valeurs trouvées étant du même ordre que celles contrôlées par PRUD'HON (I967), sur des sujets de la même souche; mais les enregistrements graphiques ont permis de préciser que la différence de consommation n'est pas due, comme c'est le cas chez le Rat (LF MAGNEN et TALLON, I966), à une augmentation de l'amplitude moyenne des repas, mais provient d'une réduction de leur fréquence au cours de la période d'éclairement, qui pourrait être étroitement associée à l'existence de phases de sommeil lent et paradoxal diurnes décrites par FAURE et al (I966).

Il serait utile d'étudier quelle peut être l'influence de la photopériode sur le comportement alimentaire du Lapin ; FAURE et al. (I966) ont montré que le rythme normal de cæcotrophie était aisément perturbé sous l'effet de synchroniseurs externes (milieu, éclairement). 
La durée totale des repas (I h 30 à 2 h I 5 par jour) est sensiblement plus faible que les valeurs citées par LEBAS (I970).

Il s'agit, il est vrai, d'observations réalisées sur des sujets ayant presque achevé leur développement; une étude de la durée quotidienne de consommation au cours de la croissance a été entreprise. Il serait utile également d'analyser l'influence de la composition et des caractéristiques des aliments sur la prise de nourriture par le Lapin.

Enfin, il convient de souligner que nous n'avons constaté aucune liaison étroite entre l'importance des repas solides et la durée des intervalles les séparant des repas qui précèdent ou qui suivent. Chez le Rat blanc, IE MaGnen et TaLLon (Ig66) ont constaté une relation entre 1'importance des repas et la durée du repos alimentaire postprandial. Il est possible que les mécanismes impliqués ne soient pas les mêmes chez le Lapin dont l'estomac est en état de quasi-réplétion permanent. Il est possible également que la cæcotrophie, non enregistrée ici, vienne modifier les phénomènes de régulation de la consommation d'aliments d'origine exogène.

\section{Reçu pour publication en mars 1972.}

\section{SUMMARY}

\section{AUTOMATIC GRAPH RECORDING OF THE SOLID AND IIQUID FEED INTAKE IN $A D$ LIBITUM FED DOMESTIC RABBITS}

A device for automatic graph recording of the liquid and solid feed intake of ad libitum fed domestic rabbits is described.

This apparatus has been used to measure the feed intake of 6 rabbits from the same litter, 3 males and 3 females, at the age of 28 to 34 weeks.

The subjects studied consumed, on average, 23 to 33 solid meals per day, lasting between I $h 30$ and $2 \mathrm{~h}$ I5, and $\mathrm{I} 3$ to I9 liquid meals.

The number, length and distribution of the meals during 24 hours are relatively repeatable from one day to another in the same animals, but fairly different from one animal to another.

About $60 \mathrm{p}$. Ioo of the consumption of water and pellets took place during the Io hours of darkness. This unevenness in the distribution was due to a higher frequency of night-meals and to more or less prolonged " eating-pauses " during the period of light.

\section{RÉFÉRENCES BIBLIOGRAPHIQUES}

Bacou F., I970. Rythme circadien de l'activité motrice chez le Poulet et le Lapin domestique. Diplôme d'études approfondies $30 \mathrm{pp}$. Montpellier.

Faure J., Vincent J.-D., Bensch C., r966. Sur des relations observées entre sommeil, rythmes alimentaires et état hormonal. Cah. Nutr. Diet., 1, I9-32.

Lebas F., I969a. Alimentation lactée et croissance pondérale du Lapin avant sevrage. Ann. Zootech., $18,197 \cdot 208$.

Lebas F., Ig69 b. L'alimentation du Lapin. L'alimentation et la vie., 57, 245-268.

LEBAS F., r97o. Aspects techniques de la production du Lapin de chair. Entreprise agricole. Janvier I970, $5 \mathrm{I}-6 \mathrm{I}$.

Lebas F., I97I. Nombre de postes de consommation pour les groupes de lapins en croissance. Bull. Tech. inf. ingrs. servs, agric. $26,56 \mathrm{I}-564$.

Le Magnen J., Tallon S., 1966. La périodicité spontanée de la prise d'aliments ad libitum du Rat blanc. J. Physiol. Paris 58, 323-349. 
PRUD,HON M., 1967. L'appétit du lapin alimenté à sec. Bull. Tech. inf. ingrs. servs. agric., 21 , I-I6. Prud'hon M., BeL L., I968. Le sevrage précoce des lapereaux et la reproduction des lapines. $A n n$. Zootech., 17, 23-30.

Rovvier R., i970. L'amélioration génétique du Lapin de chair. Revue de l'Élevage. $4 \mathbf{4}^{\text {ry }} \mathrm{n}^{\mathbf{0}}$ spécial. II3-I2I.

Worden A. N., LEAHY J. S., 1962, in HAFEZ (E. S. E.). The behaviour of domestic animals. 397-4I4, Baillière. Londres.

YARDIN M., I968. Quelques aspects qualitatifs et quantitatifs de l'activité de la lapine allaitante (Oryctolagus cunicuus). Rev. Comp. Animal., 2, 72-76.

Zarrow M. X., Debenenberg V. M., Anderson C. O., I965. Rabbit frequency of suckling in the pup. Science. 150, I835-36. 